\title{
Prognostic value of inflammatory markers determined during diagnosis in patients with sarcoidosis: chronic versus remission
}

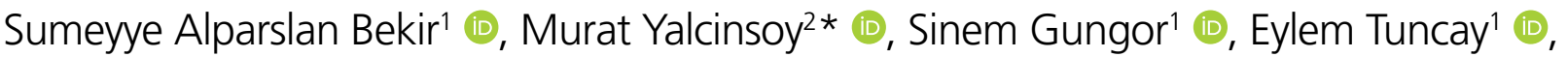

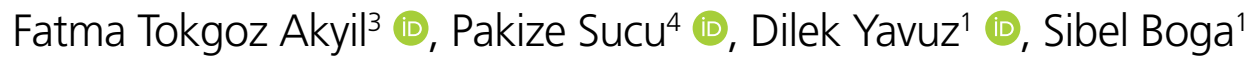

\begin{abstract}
SUMMARY
OBJECTIVE: This study aimed to evaluate the prognostic value of inflammatory markers determined during admission among patients with sarcoidosis with chronic and remission groups.

METHODS: This study was designed as retrospective single-center study. Patients with sarcoidosis without treatment and who had at least two years of follow-up were included in this study. Patients were divided into two groups as chronic and remission. The primary outcome is to evaluate hematological parameters in remission and chronic sarcoidosis groups.

RESULTS: Out of 348 patients with sarcoidosis, 142 patients without treatment and followed up for at least two years were included in this study. Groups had similar demographic features with the predominance of females ( 80.4 and $77.9 \%$, respectively) and stage I disease (78.6 and 68.6\%, respectively). Lymphocyte count [median (IQR) 1.7 (1.3-2.3) 109/L versus 2.1 (1.6-2.4) 109/L, p=0.034] was significantly lower, whereas neutrophil to lymphocyte ratio (NLR) was significantly higher [median (IQR) 2.6 (2.0-3.1) versus 2.0 (1.6-2.8), $\mathrm{p}=0.006$ ] at admission in the chronic group. No significant difference was determined in inflammatory parameters at admission between groups. CONCLUSION: Lower lymphocyte count and higher neutrophil to lymphocyte ratio were determined in patients with chronic sarcoidosis compared with the remission group, based on monitoring of radiological staging up to five-year after the initial diagnosis. Accordingly, the identification of neutrophil to lymphocyte ratio at diagnosis seems to be a potential prognostic marker in patients with sarcoidosis beside its low cost and easy determination in routine clinical practice.
\end{abstract}

KEYWORDS: Sarcoidosis. Chronic granulomatous disease. Prognosis.

\section{INTRODUCTION}

Sarcoidosis is a systemic inflammatory granulomatous disease of unknown etiology, with pulmonary involvement in most cases, and associated with heterogeneous clinical presentation and disease course ${ }^{1-4}$. Although a favorable outcome with self-limiting clinics occurs in most of the patients, progression to fibrosis and thus permanent organ impairment occurs in one-third of the patients ${ }^{5,6}$.

Several clinical, physiological, and radiographic parameters were under investigation for objective disease-specific biomarkers

\footnotetext{
'University of Health Sciences, Sureyyapasa Chest Diseases and Thoracic Surgery Training and Research Hospital, Department of Pulmonary Disease - Istanbul, Turkey.

${ }^{2}$ Inonu University, Medical Faculty, Department of Pulmonary Disease - Malatya, Turkey.

${ }^{3}$ University of Health Sciences, Yedikule Chest Diseases and Thoracic Surgery Training and Research Hospital, Department of Pulmonary Disease Istanbul, Turkey.

${ }^{4}$ Arnavutköy Public Hospital, Department of Pulmonary Disease - Istanbul, Turkey.

*Corresponding author: mrtyalcinsoy@yahoo.com

Conflicts of interest: the authors declare there are no conflicts of interest. Funding: none.

Received on August 18, 2021. Accepted on August 19, 2021.
} 
that can predict the severity and prognosis of sarcoidosis ${ }^{3,4,7,8}$. Diagnostic and prognostic roles of inflammatory markers have been addressed by several studies in inflammatory and chronic respiratory diseases ${ }^{4,5,9-14}$. Besides, limited data about serum inflammatory markers were available in the monitoring of patients with sarcoidosis s, $, 9,13,14^{\text {. }}$

Neutrophil to lymphocyte ratio (NLR) has been recently emerged as a cost-effective, potential inflammatory marker and found to be associated with the severity and prognosis of systemic inflammatory diseases, cancer, and respiratory and cardiac diseases. Although there are a limited number of studies, NLR was shown to be increased in patients with sarcoidosis as compared with the control group ${ }^{4,5,13,14}$.

Therefore, this retrospective study was designed to evaluate the prognostic value of serum inflammatory markers [i.e., NLR, mean platelet volume (MPV), platelet/MPV ratio, angiotensin-converting enzyme (ACE), and C-reactive protein (CRP)] determined at initial diagnosis in the clinical course of disease among patients with sarcoidosis.

\section{METHODS}

\section{Study population}

Patients with sarcoidosis without treatment and who had at least 2 years of follow-up were included in this retrospective study. Patients were divided into two groups, namely, chronic and remission. Figure 1 shows the flowchart of patient enrollment.

\section{Diagnosis of Sarcoidosis}

Sarcoidosis was diagnosed as if clinical and radiological data are supported by the presence of non-caseating granulomas in a biopsy specimen, and alternative causes of granulomatous inflammation are ruled out. For patients who did not allow biopsy and/or had Löfgren syndrome, sarcoidosis was diagnosed by clinical, radiological, and laboratory compatibilities.

There are five roentgenographic stages as follows: Stage 0: normal chest X-ray; Stage 1: bilateral hilar lymphadenopathy; Stage 2: bilateral hilar lymphadenopathy together with parenchymal involvement; Stage 3: parenchymal involvement without bilateral hilar lymphadenopathy; and Stage 4: pulmonary fibrosis ${ }^{1}$.

- Remission: Spontaneous clinical and radiological improvement ${ }^{15}$.

- Chronic sarcoidosis: Persistence of sarcoidosis more than 2 years $^{15}$.

- Extrapulmonary involvement: The criteria specified in A Case-Control Etiologic Study of Sarcoidosis (ACCESS) trial were used for the definition of organ involvement ${ }^{16}$.

- Hematological analysis
Complete blood count analysis was performed via flow cytometry (Beckman Coulter LH 780 Analyzer; Beckman Coulter Inc., Miami, FL, USA). Serum CRP levels were determined by using the turbidimetric method using a BN II System (Siemens, Munich, Germany). ACE (U/L) was measured by using the spectrophotometric methods (EN Biochemical Enterprise, Italy). NLR was calculated by the ratio of neutrophil to lymphocyte counts. The platelet to MPV ratio was also calculated by the ratio of the platelet count to MPV.

\section{Outcome measures}

Data on patient demographics, stage of the disease, duration of follow up, and total blood count (i.e., neutrophil, lymphocyte, and thrombocyte) and inflammatory markers (i.e., ACE, CRP, MPV, NLR, and platelet/MPV ratio) determined at diagnosis were obtained from medical records in both chronic and remission groups. The percentage of survivors and non-survivors was also recorded in both groups.

\section{Ethics}

The study was approved by the Ethical Committee of the institution (05.11.2020/116.2007.197) and was in accordance with the Declaration of Helsinki. As informed consent from patients to review their medical records was not obtained, patient data were de-identified and strictly protected.

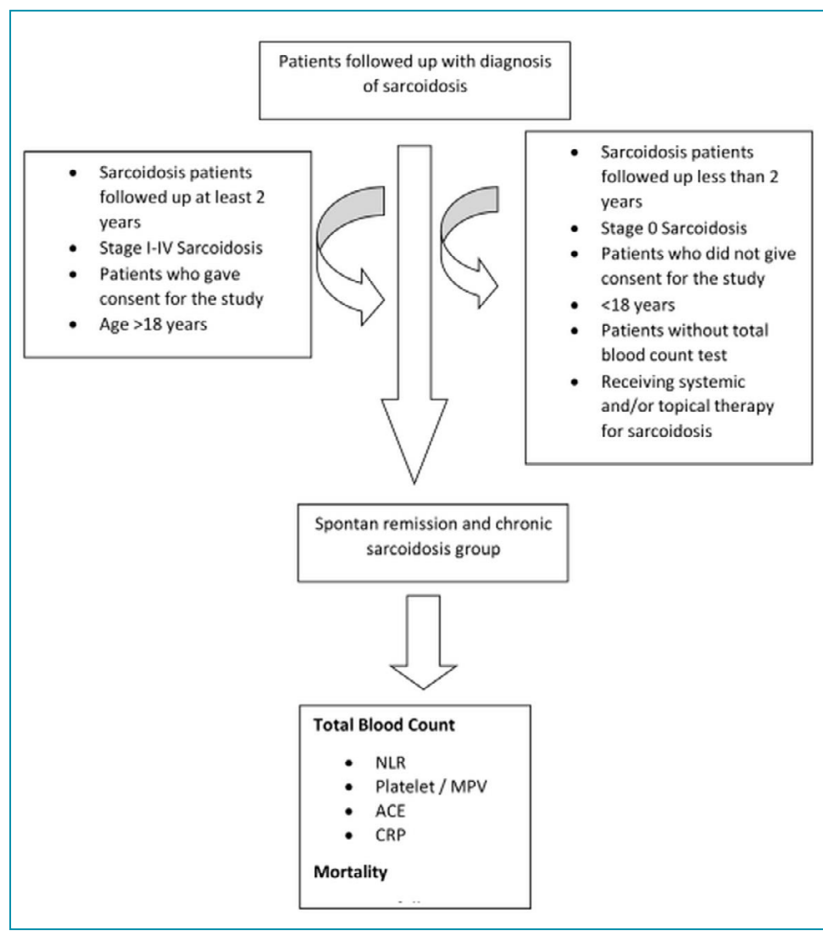

Figure 1. Flowchart of this study. 


\section{Statistical analysis}

Statistical analysis was made using the computer software Statistical Package for Social Sciences (IBM SPSS Statistics for Windows, version 21.0, released 2012, IBM Corp., Armonk, New York, USA). Fisher's exact test and Pearson's chi-square analysis were performed for categorical variables. Mann-Whitney $\mathrm{U}$ test was used for comparison of numerical variables. Data were expressed as "mean (standard deviation; SD)", "n (\%)," and "median (minimum and maximum)" values, where appropriate. $\mathrm{p}<0.05$ was considered statistically significant.

\section{RESULTS}

Among 348 patients with sarcoidosis, 142 patients with stage I-III sarcoidosis without treatment and had at least two years of follow-up were included in this retrospective study.

Remission and chronic groups had similar demographic and sarcoidosis features with the predominance of females $(80.4$ and $77.9 \%$, respectively) and Stage I disease (78.6 and 68.6\%, respectively). Stage IV sarcoidosis did not exist in this study population. Extrapulmonary involvement was determined in one-third of patients in both groups. Remission occurred in $39.4 \%$ of patients (Table 1 ).

Patients in the remission and chronic groups had a similar duration of follow-up (median 62.5 and 60.0 months, respectively) and low mortality (0.0 and 4.7\%, respectively; Table 1$)$.

Lymphocyte count [median (IQR) $1.7(1.3-2.3) 10^{9} / \mathrm{L}$ versus $\left.2.1(1.6-2.4) 10^{\%} / \mathrm{L}, \mathrm{p}=0.034\right]$ was significantly lower, whereas
NLR was significantly higher [median (IQR) 2.6 (2.0-3.1) versus 2.0 (1.6-2.8), $\mathrm{p}=0.006$ ] at admission in the chronic group when compared with remission group. No significant difference was noted in other inflammatory parameters between groups including ACE, CRP, MPV, and PLT/MPV ratio (Table 2).

\section{DISCUSSION}

In this study, despite the similar baseline demographic and disease characteristics, the chronic sarcoidosis group had decreased lymphocyte count and increased NLR, compared with the remission group. Lymphocyte count and NLR levels were found to indicate the prognosis in patients with sarcoidosis. Thus, patients with low lymphocyte count and high NLR values had a higher likelihood of developing chronic disease than the clinical remission.

Sarcoidosis is observed most frequently in women in different studies ${ }^{17,18}$. In this study, the ratios of female patients were similar in both groups. In recent studies, the ratios of patients with stages I and II were higher in all patients with sarcoidosis, which is compatible with the literature ${ }^{17-19}$. In this study, patients with stage IV sarcoidosis did not exist, besides the ratio of stage II disease in the chronic group was almost two-fold greater than the remission group without statistical significance.

In recent studies, remission in sarcoidosis was reported to occur in more than half of patients within 3 years ${ }^{6,7}$. In a study conducted by Scadding et al., complete radiological remission after $>5$-year follow-up was reported to occur in $84 \%, 58 \%$,

Table 1. Demographic and disease-related characteristics in study groups.

\begin{tabular}{|c|c|c|c|c|}
\hline & & Remission $(n=56)$ & Chronic course $(n=86)$ & $p$-value \\
\hline \multirow{2}{*}{ Age (year) } & Mean (SD) & $41.5(11.8)$ & $44.1(12.3)$ & \multirow{2}{*}{$0.174^{a}$} \\
\hline & Median (IQR) & $42.0(31.3-51.8)$ & $47.0(34.0-52.3)$ & \\
\hline \multicolumn{5}{|c|}{ Gender, n (\%) } \\
\hline Female & & $45(80.4)$ & $67(77.9)$ & \multirow{2}{*}{$0.448^{b}$} \\
\hline Male & & $11(19.6)$ & $19(22.1)$ & \\
\hline \multicolumn{5}{|c|}{ Disease stage } \\
\hline Stage I & & $44(78.6)$ & $59(68.6)$ & \multirow{3}{*}{$0.425^{b}$} \\
\hline Stage II & & $10(17.9)$ & $23(26.7)$ & \\
\hline Stage III & & $2(3.6)$ & $4(4.7)$ & \\
\hline Extrapulmo & & $19(33.9)$ & $25(29.1)$ & $0.334^{b}$ \\
\hline Duration of & th), median (IQR) & $62.5(32.5-90.8)$ & $60.0(41.3-120.0)$ & $0.393^{a}$ \\
\hline \multicolumn{5}{|c|}{ Survival, n (\%) } \\
\hline Died & & $0(0.0)$ & $4(4.7)$ & \multirow{2}{*}{$0.131^{b}$} \\
\hline Survived & & $56(100.0)$ & $82(95.3)$ & \\
\hline
\end{tabular}

aMann-Whitney $U$ test; ${ }^{b} \chi^{2}$ test. 
Table 2. Hematological parameters in study groups.

\begin{tabular}{l|c|c|c} 
& Remission $(n=56)$ & Chronic course $(n=86)$ & p-value $^{a^{2}}$ \\
\hline ACE & $53.0(35.5-82.8)$ & $56.0(32.8-96.0)$ & 0.666 \\
\hline Neutrophil $\left(10^{9} / \mathrm{L}\right)$ & $4.1(3.2-5.07)$ & $4.3(3.5-5.4)$ & 0.277 \\
\hline Lymphocyte $\left(10^{9} / \mathrm{L}\right)$ & $2.1(1.6-2.4)$ & $1.7(1.3-2.3)$ & 0.034 \\
\hline NLR & $2.0(1.6-2.8)$ & $2.6(2.0-3.1)$ & 0.006 \\
\hline Platelet $\left(10^{9} / \mathrm{L}\right)$ & $271.0(220.3-308.6)$ & $256.3(216.8-317.0)$ & 0.757 \\
\hline MPV (fL) & $8.6(7.7-9.2)$ & $8.5(7.9-9.0)$ & 0.784 \\
\hline Platelet/MPV & $31.2(24.3-38.1)$ & $30.6(25.9-38.4)$ & 0.948 \\
\hline CRPb & $3.5(3.0-16.5)$ & $12.5(4.5-26.5)$ & 0.228 \\
\hline
\end{tabular}

Data are shown as median (IQR). ${ }^{a}$ Mann-Whitney U test; based on data from 12 patients in the remission and 10 patients in the chronic groups. ACE: angiotensin-converting enzyme; CRP: C-reactive protein; MPV: mean platelet volume; NLR: neutrophil to lymphocyte ratio. Numbers in bold are statistically significant.

and $10-20 \%$ of patients with initial radiological stages I, II, and III, respectively ${ }^{20}$. Besides, in this study, the ratio of the patients with chronic sarcoidosis was nearly $25 \%$, which was mentioned $10-30 \%$ in different studies ${ }^{20,21}$.

Hematological changes in sarcoidosis include mild anemia, leukocytosis, leukopenia, lymphopenia, eosinophilia, and thrombocytopenia, which were frequently observed in recent studies $^{4,22}$. Notably, in this study compared with the remission group, the chronic group had a lower lymphocyte count, while a similar neutrophil count was determined during the diagnosis. This seems to indicate the prominent role of lymphocytopenia, as a marker of physiological oxidative stress.

The NLR is a simple composite marker providing the objective measurement of changes in both neutrophils and lymphocytes, while calculated easily from a routine complete blood count without needing extra effort or $\operatorname{cost}^{4,5,13}$. Hence, in this study, the presence of high NLR during diagnosis as a poor prognostic marker seems notable with at least 5-year follow-up.

The NLR is novel prognostic, diagnostic marker for inflammatory diseases ${ }^{11,13,14,23}$. In the study by İliaz et al., the NLR was found to be supportive in differentiation between tuberculosis and sarcoidosis with the cutoff value of $2.55^{13}$. In a study by Dirican et al., NLR values were reported to be higher in patients with sarcoidosis than in healthy controls and in extrapulmonary involvement ${ }^{5}$. In a large-scale study by Gungor et al. among patients with sarcoidosis, high NLR $(\geq 2.0)$ values have demonstrated the correlation with well-known inflammatory markers such as ACE, erythrocyte sedimentation rate (ESR), and $\mathrm{CRP}^{14}$. In this study, since there are limited data about the role of NLR in the chronic sarcoidosis and remission group, the NLR was significantly higher at admission in the chronic sarcoidosis.
Patients with sarcoidosis with stage II and III disease and extrapulmonary involvement were reported to be more likely to have a high NLR 5 . Despite significantly higher NLR in the chronic group in this study, two groups had a similar NLR in terms of initial disease stage and extrapulmonary involvement. Thus, in this study, baseline similarity of disease characteristics between two clinical outcome groups further emphasizes the need for potential biomarkers in sarcoidosis that could predict the disease-specific prognosis following the initial diagnosis.

In recent studies, MPV values were found to be higher in patients with sarcoidosis than in the control group ${ }^{5,24}$. While the stage increases, no changes in MPV values were detected in the study by Dirican et al. ${ }^{5}$. In contrast, in this study, no significant difference was found between the two groups according to MPV and PLT/MPV values. While serum ACE is the most widely used biomarker of diagnostic and prognostic values in patients with sarcoidosis, it has been associated with relatively low specificity, as elevated serum ACE activity was also shown in multiple granulomatous and non-granulomatous diseases. ACE was also shown to be insignificant in determining the severity of the disease and inappropriate to be used during follow-up'. In this study, there was no significant change in serum ACE values measured at the time of admission, between chronic and remission groups.

In recent studies, CRP levels were found to be useful to predict the effectiveness of treatment in chronic pulmonary sarcoidosis. Besides, studies have also analyzed the usefulness of CRP levels reporting higher significant levels with respect to healthy controls ${ }^{25}$. In this study, according to CRP values, no significant difference was determined between the two groups.

Both stage III sarcoidosis and extrapulmonary involvement were reported to be associated with chronic and progressive diseases and increased the likelihood of relapse ${ }^{20}$. In this study, 
the mortality rate in the chronic group (4.7\%) was similar to the meta-analysis designed by Reich et al. (4.8\%). The ratio of stage III disease (4.7\%) in this study is related to the mortality rate $(4.7 \%)$.

The major limitation of this study is its retrospective single-center design and relatively low sample size due to the rarity of the disease, which makes our findings not generalizable to the general patient population with sarcoidosis. As patients were asymptomatic in both groups, a measurable outcome in terms of clinical implications of NLR might add strength to the study. Since the data on the prognostic role of inflammatory markers in sarcoidosis are not well defined, our findings make a valuable contribution to the literature.

\section{CONCLUSION}

This study demonstrated that the chronic sarcoidosis group had a higher NLR value and lower lymphocyte count at diagnosis compared with the remission group in long-term follow-up. Therefore, NLR values obtained during diagnosis seem to have prognostic value in the long-term monitoring of patients with sarcoidosis in routine clinical practice. The similarity between chronic and remission groups in terms of initial disease stage and prevalence of extrapulmonary involvement, this study emphasizes the importance of developing novel serum biomarkers to offer a reliable and objective measure to predict disease-specific prognosis among patients with sarcoidosis. Future studies in larger series are needed to justify the diagnostic and prognostic significance of inflammatory markers in patients with sarcoidosis.

\section{AUTHORS' CONTRIBUTIONS}

SAB: Conceptualization, Data curation, Formal analysis, Writing - original draft, Writing - review \& editing. MY: Conceptualization, Data curation, Writing - original draft, writing - review \& editing. SG: Conceptualization, Data curation, Writing - original draft, writing - review \& editing. ET: Investigation, Methodology, Resources, Formal analysis, Writing - original draft, Writing - review \& editing. FTA: Data curation, Formal analysis, Methodology, Resources, Writing original draft. PS: Conceptualization, Data curation, Resources, Writing - review \& editing. DY: Data curation, Investigation, Resources, Visualization, Writing - original draft. SB: Formal analysis, Investigation, Resources, Writing - review \& editing.

\section{REFERENCES}

1. Society AT. Statement on sarcoidosis. Joint Statement of the American Thoracic Society (ATS), the European Respiratory Society (ERS) and the World Association of Sarcoidosis and Other Granulomatous Disorders (WASOG) adopted by the ATS Board of Directors and by the ERS Executive Committee, February 1999. Am J Respir Crit Care Med. 1999;160(2):736-55. https://doi.org/10.1164/ ajrccm.160.2.ats4-99

2. Kıter G, Müsellim B, Cetinkaya E, Türker H, Uzaslan AK, Yentürk E, et al. Clinical presentations and diagnostic work-up in sarcoidosis: a series of Turkish cases (clinics and diagnosis of sarcoidosis). Tuberk Toraks. 2011;59(3):248-58. PMID: 22087521

3. Ahmadzai H, Loke WSJ, Huang S, Herbert C, Wakefield D, Thomas PS. Biomarkers in sarcoidosis: a review. Curr Biomark Find. 2014;4:93-106. https://doi.org/10.2147/CBF.S46196

4. Ocal N, Dogan D, Ocal R, Tozkoparan E, Deniz O, Ucar E, et al. Effects of radiological extent on neutrophil/lymphocyte ratio in pulmonary sarcoidosis. Eur Rev Med Pharmacol Sci. 2016;20(4):709-14. PMID: 26957274

5. Dirican N, Anar C, Kaya S, Bircan HA, Colar HH, Cakir M. The clinical significance of hematologic parameters in patients with sarcoidosis. Clin Respir J. 2016;10(1):32-9. https://doi. org/10.1111/crj.12178

6. Iannuzzi MC, Rybicki BA, Teirstein AS. Sarcoidosis. N Engl J Med. 2007;357(21):2153-65. https://doi.org/10.1056/ NEJMra071714

7. Casanova N, Zhou T, Knox KS, Garcia JGN. Identifying Novel Biomarkers in Sarcoidosis Using Genome-Based Approaches.
Clin Chest Med. 2015;36(4):621-30. https://doi.org/10.1016/j. ccm.2015.08.005

8. Mañá J, Salazar A, Manresa F. Clinical factors predicting persistence of activity in sarcoidosis: a multivariate analysis of 193 cases. Respiration. 1994;61(4):219-25. https://doi. org/10.1159/000196341

9. Rothkrantz-Kos S, van Dieijen-Visser MP, Mulder PG, Drent $M$. Potential usefulness of inflammatory markers to monitor respiratory functional impairment in sarcoidosis. Clin Chem. 2003;49(9):1510-7. https://doi.org/10.1373/49.9.1510

10. Chen G, Wu C, Luo Z, Teng Y, Mao S. Platelet-lymphocyte ratios: a potential marker for pulmonary tuberculosis diagnosis in COPD patients. Int J Chron Obstruct Pulmon Dis. 2016;11:2737-40. https://doi.org/10.2147/COPD.S111254

11. Yoon NB, Son C, Um SJ. Role of the neutrophil-lymphocyte count ratio in the differential diagnosis between pulmonary tuberculosis and bacterial community-acquired pneumonia. Ann Lab Med. 2013;33(2):105-10. https://doi.org/10.3343/ alm.2013.33.2.105

12. Paone G, Leone V, Conti V, De Marchis L, Ialleni E, Graziani C, et al. Blood and sputum biomarkers in COPD and asthma: a review. Eur Rev Med Pharmacol Sci. 2016;20(4):698-708. PMID: 26957273

13. Iliaz S, Iliaz R, Ortakoylu G, Bahadir A, Bagci BA, Caglar $E$. Value of neutrophil/lymphocyte ratio in the differential diagnosis of sarcoidosis and tuberculosis. Ann Thorac Med. 2014;9(4):232-5. https://doi.org/10.4103/1817-1737.140135

14. Gungor S, Akturk UA, Yalcinsoy M, Kocak ND, Goksenoglu NC, Altunbey SA, et al. What is the neutrophil/lymphocyte 
ratio in sarcoidosis? Bratisl Lek Listy. 2016;117(3):152-5. https://doi.org/10.4149/bll_2016_030

15. Hunninghake GW, Costabel U, Ando M, Baughman R, Cordier JF, du Bois R, et al. A ATS/ERS/WASOG statement on sarcoidosis. American Thoracic Society/European Respiratory Society/World Association of Sarcoidosis and other Granulomatous Disorders. Sarcoidosis Vasc Diffuse Lung Dis. 1999;16(2):149-73. PMID: 10560120

16. Design of a case control etiologic study of sarcoidosis (ACCESS). ACCESS Research Group. J Clin Epidemiol. 1999;52(12):117386. https://doi.org/10.1016/s0895-4356(99)00142-0

17. Lill $\mathrm{H}$, Kliiman $\mathrm{K}$, Altraja A. Factors signifying gender differences in clinical presentation of sarcoidosis among Estonian population. Clin Respir J. 2016;10(3):282-90. https://doi.org/10.1111/crj.12213

18. Mañá J, Rubio-Rivas M, Villalba N, Marcoval J, Iriarte A, Molina-Molina $M$, et al. Multidisciplinary approach and longterm follow-up in a series of 640 consecutive patients with sarcoidosis: Cohort study of a 40-year clinical experience at a tertiary referral center in Barcelona, Spain. Medicine (Baltimore). 2017;96(29):e7595. https://doi.org/10.1097/ MD.000000000000759519

19. Pérez-Alvarez R, Brito-Zerón P, Kostov B, Feijoo-Massó $C$, Fraile G, Gómez-de-la-Torre R, et al. Systemic phenotype of sarcoidosis associated with radiological stages. Analysis of 1230 patients. Eur J Intern Med. 2019;69:77-85. https://doi. org/10.1016/j.ejim.2019.08.025
20. Scadding JG. Prognosis of intrathoracic sarcoidosis in England. A review of 136 cases after five years' observation. Br Med J. 1961;2(5261):1165-72. https://doi.org/10.1136/ bmj.2.5261.1165

21. Hillerdal G, Nöu E, Osterman K, Schmekel B. Sarcoidosis: epidemiology and prognosis. A 15-year European study. Am Rev Respir Dis. 1984;130(1):29-32. https://doi.org/10.1164/ arrd.1984.130.1.29

22. Gupta D, Rao VM, Aggarwal AN, Garewal G, Jindal SK. Haematological abnormalities in patients of sarcoidosis. Indian J Chest Dis Allied Sci. 2002;44(4):233-6. PMID: 12437235

23. Dirican A, Ekinci N, Avci A, Akyol M, Alacacioglu A, Kucukzeybek $Y$, et al. The effects of hematological parameters and tumorinfiltrating lymphocytes on prognosis in patients with gastric cancer. Cancer Biomark. 2013;13(1):11-20. https://doi. org/10.3233/CBM-130331

24. Kemal CT, Aylin OA, Volkan K, Seda M, Recep B, Can S. The importance of PET/CT findings and hematological parameters in prediction of progression in sarcoidosis cases. Sarcoidosis Vasc Diffuse Lung Dis. 2017;34(3):242-50. https://doi.org/10.36141/ svdld.v34i3.5299

25. Ramos-Casals M, Retamozo S, Sisó-Almirall A, Pérez-Alvarez $R$, Pallarés L, Brito-Zerón P. Clinically-useful serum biomarkers for diagnosis and prognosis of sarcoidosis. Expert Rev Clin Immunol. 2019;15(4):391-405. https://doi.org/10.1080/174 4666X.2019.1568240 